\title{
Broad Band Equilibration of Strangeness
}

Gerald. E. Brown ${ }^{\mathrm{a}}$, Mannque Rho ${ }^{\mathrm{b}}$ and Chaejun Songa*

${ }^{a}$ Department of Physics and Astronomy, State University of New York

Stony Brook, NY 11794-3800, USA

bService de Physique Théorique, CE Saclay, 91191 Gif-sur-Yvette, France

We develop the "broad band equilibration" scenario for kaon productions at GSI energies with in-medium effects.

\section{Introduction}

As shown in some talks [1] of this conference, statistical descriptions work very successfully for multihadron final states in nucleon-nucleon, nucleon-nucleus, and nucleus-nucleus collisions. In this approach the chemical equilibrium particle ratios are functions of temperature $T$ and baryon chemical potential $\mu_{B}$ only

Recently GSI measured Au-Au and Ni-Ni collisions at $0.8 \sim 2.0 \mathrm{GeV}$. Some measured particle multiplicity ratios are in Table 1 below. Cleymans, Oeschler and Redlich [2] fit

Table 1

Experimental results for different particle ratio in central $\mathrm{Ni}+\mathrm{Ni}$ collisions.

\begin{tabular}{ccccc}
\hline$\pi^{+} / p$ & $K^{+} / \pi^{+}$ & $\pi^{-} / \pi^{+}$ & $d / p$ & $K^{+} / K^{-}$ \\
\hline 0.17 & 0.0084 & 1.05 & 0.28 & 32 \\
\hline
\end{tabular}

All the data except for $K^{+} / K^{-}$comes from [2] at $E=1.8 \mathrm{~A} \mathrm{GeV} \cdot K^{+} / K^{-}$comes from [3] at $E=1.93 \mathrm{~A} \mathrm{GeV}$.

them well by a common chemical freezeout at $T=70 \mathrm{MeV}$ and $\mu_{B}=750 \mathrm{MeV}$ in terms of a canonical thermal model. This is another success of statistical description. However, it is hard to understand that the chemical freezeout density from these values of $T$ and $\mu_{B}$ is just $\rho_{0} / 4$ where the $K^{-}$mean free path is $\sim 6 \mathrm{fm}$. Bratkovskaya and Cassing's transport calculations in Fig. 1 1 show that $K^{-}$production comes from all densities.

We can understand simply why they are compelled to obtain such a low density. By strangeness conservation the number of $K^{+}$is the sum of the number of $K^{-}$and that of $\Lambda$ f

${ }^{*}$ GEB and CS were supported by the US Department of Energy under Grant No. DE-FG02-88 ER40388. ${ }^{\dagger}$ In the canonical description for heavy ion collision we need a system size parameter but the parameters are related to the number of participants. For example, $V \sim 1.9 \pi A_{p}$ [2]. And in the grand canonical description we need the chemical potentials of conserved charges.

${ }^{\ddagger}$ Of course we need to include $\Sigma$ and $\Xi$ hyperon. Here they are neglected to simplify the discussion. 


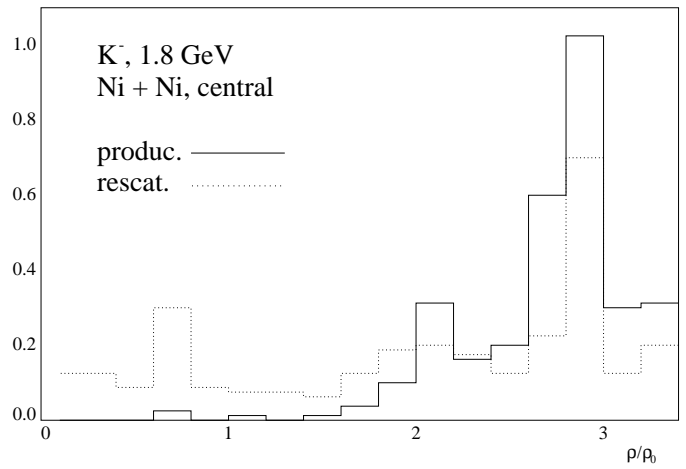

Figure 1. Bratkovskaya and Cassing's calculations of $K^{-}$production (private communication). The solid line and dashed line give the density of the origin and that of the last interaction, respectively.

We measured $K^{+} / K^{-} \sim 32$ in Table 1 so $\Lambda / K^{-} \sim 31$. Thus if we get the right $\Lambda / K^{-}$ratio, $K^{+} / K^{-}$follows. The ratio is

$R=\frac{\Lambda}{K^{-}}=\left(\frac{\bar{p}_{\Lambda}}{\bar{p}_{\bar{K}}}\right)^{\frac{3}{2}} \frac{e^{-\left(E_{\Lambda}-\mu_{B}\right) / T}}{e^{-E_{\bar{K}} / T}} \approx\left(\frac{M_{\Lambda}}{M_{\bar{K}}}\right)^{\frac{3}{2}} \frac{e^{-\left(M_{\Lambda}-\mu_{B}\right) / T}}{e^{-M_{\bar{K}} / T}}$.

If $\mu_{B}=M_{N}, M_{\Lambda}=M_{N}+175 \mathrm{MeV}$ gives $R \sim 280$. It's too large so they need $\mu_{B}=750$ $\mathrm{MeV}, 190 \mathrm{MeV}$ lower than $M_{N}$, in order to get the right value of $R$. That's why they obtained the low density for equilibration with free-space mass hadrons.

\section{In-medium kaons}

A simple way to introduce an in-medium $K^{-}$mass is the $\mathrm{V}$-spin formalism in [4]. In the formalism we can obtain the kaon effective mass by considering the kaon fields as small fluctuations $\theta$ around the $\sigma$ axis as shown in Fig. 2. The Hamiltonian for explicit chiral symmetry breaking is

$H_{X \chi S B}=\Sigma_{K N}<\bar{N} N>\cos \theta+\frac{1}{2} m_{K}^{2} f^{\star 2} \sin ^{2} \theta$.

Then the effective mass of kaons (small fluctuation $\theta$ ) comes to drop from movement towards restoration of explicitly broken chiral symmetry;

$\frac{m_{K}^{\star 2}}{m_{K}^{2}}=1-\frac{\Sigma_{K N} \rho}{f^{\star 2} m_{K}^{2}}$

with an approximation $\left\langle\bar{N} N>\approx \rho\right.$. Taking $m_{s} \sim 150 \mathrm{MeV}, m_{u}=m_{d}=6 \mathrm{MeV}$, $\Sigma_{\pi N}=46 \mathrm{MeV}$ and $<N|\bar{s} s| N>\sim \frac{1}{3}<N|\bar{d} d| N>$ [5], we obtain

$\Sigma_{K N}=\frac{\left(m_{u}+m_{s}\right)<N|\bar{u} u+\bar{s} s| N>}{\left(m_{u}+m_{d}\right)<N|\bar{u} u+\bar{d} d| N>} \Sigma_{\pi N} \sim 400 \mathrm{MeV}$. 


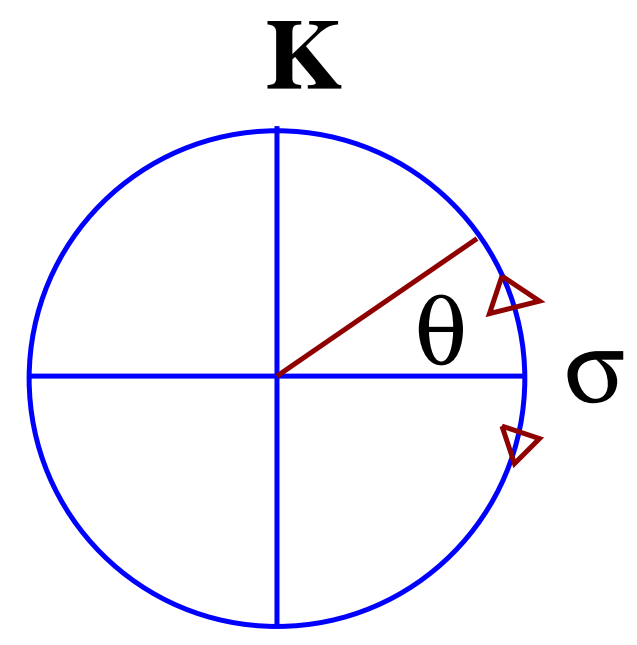

Figure 2. V-formalism

The effective value of $\Sigma_{K N}$ is somewhat smaller by range corrections [6];

$\left(\Sigma_{K N}\right)_{e f f}=\left(1-0.37 \omega_{K}^{\star 2} / m_{K}^{2}\right) \Sigma_{K N}$

where $\omega_{K^{-}}^{\star}=m_{K^{-}}-\frac{\omega_{K}^{\star}}{m_{K}} V_{K}$ is the self-energy of the antikaon at rest with vector potential $V_{K}$.

\section{Broad equilibration and kaon condensation}

Considering kaon mass shift in medium, we can rewrite $R$ in Eq. (11) as

$R=\left(\frac{M_{\Lambda}}{m_{K^{-}}^{\star}}\right)^{3 / 2} e^{\left(\mu_{B}+\omega_{K^{-}}^{\star}\right) / T} e^{-M_{\Lambda} / T}$.

We assume that kaon vector potential $V_{K}$ is proportional to the density below $\rho_{0}$ and constant above $\rho_{0}$, because the vector decoupling is expected at high density [7]. As $\mu_{B}$ goes from $860 \mathrm{MeV}$ to $905 \mathrm{MeV}, \rho$ goes from $1.2 \rho_{0}$ to $2.1 \rho_{0}$ and $\mu_{B}+\omega_{K^{-}}^{\star}$ goes from 1230 to $1227 \mathrm{MeV}$ with fixed $T=70 \mathrm{MeV}$. Even at $\rho_{0} / 4 \mu_{B}+\omega_{K^{-}}^{\star}$ is $1245 \mathrm{MeV}$. Disregarding the dependence on $m_{K^{-}}^{\star}$ in the denominator and on $\mathrm{T}$ f for simplicity, we found the ratio $R$ is independent of density. This results can be checked by the recent experimental results. The ratio $K^{+} / K^{-}$, which is directly related to $\Lambda / K^{-}$, is almost independent of centrality in GSI [3] and AGS [8] experiments. In other words, the ratio of hyperon to $K^{-}$is roughly the same at all densities. We call it "broad-band equilibration".

So the negatively charged strangeness sector looks well and truly equilibrated within itself. Positively charged strangeness hardly changes as the system expands below $2 \rho_{0}$ [9]. Since the number of positively charged strangeness is the same as that of the negatively charged strangensess, most of the negatively charged strangeness particles will be produced early.

\footnotetext{
${ }^{\S}$ The increase of $R$ by $m_{K^{-}}^{\star}$ in the denominator can be compensated for by increasing $T$.
} 
The strong attraction that the $K^{-}$experiences in dense matter can make the kaon energy come down to the electron chemical potential and kaons be able to replace electrons. If the vector decouples in the changeover from nucleons to constituent quarks as variables as we expect, the kaon will condensate before chiral restoration. The kaon condensation sets the maximum neutron star mass $1.5 M_{\odot}$ [10]. This is consistent with the observation that all the neutron star masses are below $1.5 M_{\odot}$, in systems with degenerate companions, where the companion structure should not influence the measurement.

\section{REFERENCES}

1. R. Stock, "The dynamics of A+A collisions"; D. Rischke, "Critical review on the extraction of freezeout parameters"; K. Redlich, "Critical review of strangeness as a QGP signature"; L. Bravina, "Chemical freeze-out parameters at RHIC from the microscopic model calculations".

2. J. Cleymans, H. Oeschler and K. Redlich, Phys. Rev. C 59 (1999) 1663; Phys. Lett. 485 (2000) 27.

3. M. Menzel et al., Phys. Lett.B 495 (2000) 26; M. Menzel, University of Marburg PhD thesis, 2000

4. G.E. Brown, K. Kubodera and M. Rho, Phys. Lett. B192 (1987) 273.

5. K. F. Liu, S. J. Dong, T. Draper, D. Leinweber, J. Sloan, W. Wilcox and R. M. Woloshyn, Phys. Rev. D 59 (1999) 112001.

6. C.-H. Lee, Phys. Rep. 275 (1996) 256.

7. G.E. Brown and M. Rho, Phys. Rept. 269 (1996) 217; C. Song, G.E. Brown, D.-P. Min and M. Rho, Phys. Rev. C 56 (1997) 2244; Y. Kim and H. Lee, Phys. Rev. C 62 (2000) 037901; P.K. Sahu, W. Cassing, U. Mosel and A. Ohnishi, Nucl. Phys. bf A672 (2000) 376; G.E. Brown and M. Rho, E-print hep-ph/0103102.

8. J. Dunlop, MIT PhD thesis, 1999; L. Ahle et al., Phys. Lett. B490 (2000) 53.

9. G.E. Brown, C.M. Ko, Z.G. Wu and L.H. Xia, Phys. Rev. C43 (1991) 1881.

10. G. Q. Li, C. H. Lee and G. E. Brown, Phys. Rev. Lett. 79 (1997) 5214. 